\title{
In vivo and ex vivo virtual biopsy of the liver with near-infrared, reflectance confocal microscopy
}

\author{
Vanessa Campo-Ruiz ${ }^{1,3}$, Gregory Y Lauwers², R Rox Anderson ${ }^{1}$ Emilio Delgado-Baeza ${ }^{3}$ and \\ Salvador González ${ }^{1}$ \\ ${ }^{1}$ Wellman Laboratories of Photomedicine, Massachusetts General Hospital, Harvard University, Boston, \\ MA, USA; ${ }^{2}$ Gastrointestinal Pathology Service, Department of Pathology, Massachusetts General Hospital, \\ Harvard University, Boston, MA, USA and ${ }^{3}$ Department of Morphology-Histology, School of Medicine, \\ Universidad Autónoma de Madrid, Madrid, Spain
}

\begin{abstract}
The assessment of liver architecture is an essential part of the understanding of its physiology and pathology. Current fluorescence confocal microscopy methods face numerous drawbacks, such as cytotoxicity, quenching effect, potential negative ino- and chrono-tropic effects and leaking of fluorescent agents through the sinusoid fenestrations. The recently developed, near-infrared reflectance confocal microscopy allows highresolution optical sectioning through intact tissues, without employing fluorescent stains, while contrast between structures is provided by the natural refractivity of the tissue. The aim of this study is to assess the utility of near-infrared reflectance confocal microscopy in the evaluation of the hepatic microscopic architecture in vivo and ex vivo. Rat livers were noninvasively examined in vivo and ex vivo with near-infrared reflectance confocal microscopy. Two experimental contrast agents were subsequently used to enhance particular structures. Parenchymal and vascular structures are readily identified, as well as some intracellular details. Differences between in vivo and ex vivo states were also observed. The use of contrast agents also highlights certain morphologic structures. In conclusion, near-infrared reflectance confocal microscopy stands as a useful adjunct technique to the study of hepatic parenchyma offering details equivalent to, if not surpassing traditional light microscopy.
\end{abstract}

Modern Pathology (2005) 18, 290-300, advance online publication, 22 October 2004; doi:10.1038/modpathol.3800303

Keywords: liver; hepatocyte; in vivo; laser confocal microscopy

The assessment of tissue architecture and its alterations is an essential part of the understanding of the physiology and diseases of human organs. The time tested classical microscopic techniques remain the gold standard, although they are associated with major limitations. First, the studies and their conclusions are limited to the observations made on tissues in the ex vivo status. Second, routine histology processing is time-consuming, induces tissue artifacts, and may prevent further evaluations once the tissue has been fixed. These arguments have justified a growing interest on confocal microscopy, a technique that allows virtual high-resolution sectioning of nontransparent tissues in thin

Correspondence: Dr GY Lauwers, MD, Gastrointestinal Pathology Service, Department of Pathology, Massachusetts General Hospital, 55 Fruit Street (WRN 2), Boston, MA 02114-2696, USA. E-mail: glauwers@partners.org

Received 24 May 2004; revised and accepted 12 August 2004; published online 22 October 2004 planes. ${ }^{1}$ Currently employed fluorescent confocal microscopy allows labeling of specific structures, but faces drawbacks with regard to in vivo studies. These include cytotoxicity due to the generation and release of free radicals, photothermal damage, potential negative ino- and chronotropic effects on vessels and, in the case of organs like the liver, leaking of fluorescent agents through the sinusoidal fenestrations. In addition, the use of fluorescent agents exposes tissues to exogenous agents potentially impairing further assays, while the photobleaching effect forces the investigators to perform image acquisition rapidly.

Near-infrared reflectance confocal microscopy is a novel technique that allows high-resolution optical sectioning through intact tissues without employing exogenous fluorescent stains., ${ }^{2,3}$ In near-infrared, reflectance confocal microscopy-produced images, contrast between structures is achieved by detecting the natural differences in refractivity when they are illuminated with a laser of the near-infrared 
spectrum. Based on their optical interaction with the laser, structures are visualized in shades of black and white. By convention, hyper-refractile structures appear white on screen, whereas hypo-refractile ones are black. Images are obtained in real-time (20 frames/s) and no tissue damage is observed, since the laser is shone at a very low power (less than $30 \mathrm{~mW}$ ). The organs can thus be studied in vivo, noninvasively and to a depth of $300 \mu \mathrm{m}$ below their surface.

The aim of this study is to assess the utility of near-infrared, reflectance confocal microscopy in the study of the liver parenchyma, both in vivo and ex vivo. For this purpose, images from a series of rat livers studied with near-infrared, reflectance confocal microscopy in vivo and ex vivo are compared to those obtained from paraffin-embedded, stained sections examined under standard light microscopy.

\section{Materials and methods}

\section{Animals}

Five rats (Munich-Fromter-Wistar, male, 200-350 g, Harlan Laboratories, Indianapolis, IN, USA) were studied in vivo and ex vivo. The present study was approved by the institutional Animal Care and Ethic Committee and carried out in observance of the NIH Guidelines for the Care and Use of Laboratory Animals. Rats were kept under standard caging, feeding and light conditions until experimentation day.

\section{In Vivo Procedures}

On the day of imaging, rats were anesthetized by intramuscular injection of ketamine $(75 \mathrm{mg} / \mathrm{kg}$, Ketamine $\mathrm{HCl}$ injectable, Ben Venue Laboratories Inc., Bedford, OH, USA) and xylazine $(7 \mathrm{mg} / \mathrm{kg}$, AnaSed injectable, Lloyd Laboratories, Shanendoah, IO, USA). Once anesthetized and with a permeable airway, animals were immobilized on an ad hocdesigned board in supine decubitus position and underwent a central laparotomy from the xyphoid apophysis to the pubic symphysis. Abdominal walls were smoothly retracted sideways so as to expose the frontal lobe of the liver to the confocal microscope objective lens. In vivo near-infrared, reflectance confocal microscopy imaging was carried out within the first hour under anesthesia. The liver was constantly hydrated by manual dripping of phosphate-buffered saline (PBS; Dulbecco's phosphate buffered salt solution, $1 \times$. Sigma Pharmaceuticals, St Louis, MO, USA) at room temperature $\left(20^{\circ} \mathrm{C}\right)$. Particular care was taken as not to exert any pressure on the liver and thus induce ischemia. The liver was scanned at different points in time in three dimensions. Representative images of each specimen were captured and digitally stored.

\section{Ex Vivo Procedures}

Upon completion of in vivo studies, animals were killed by overdose of the anesthetics formerly employed. Livers were resected, kept in vials with PBS and immediately studied ex vivo. Ex vivo studies comprised near-infrared, reflectance confocal microscopy and light microscopy studies. Near-infrared, reflectance confocal microscopy studies were carried out throughout the first 30 min of the killing, both in bulk and after being sectioned with scalpel blades. Acetic acid (Heinz-brand table vinegar) and sucrose aqueous solutions (gradient solutions of table sugar dissolved in water, ranging from 20 to $35 \%$ ) were used as experimental contrast-enhancing agents for near-infrared, reflectance confocal microscopy imaging. Representative near-infrared, reflectance confocal microscopy images of each specimen were captured and digitally stored. Upon completion of ex vivo nearinfrared, reflectance confocal microscopy evaluation, liver specimens were fixed in formalin $(10 \%$ buffered formalin phosphate SF-100-4, Fisher Scientific. Fisher Chemical Company, Pittsburgh, PA, USA), embedded in paraffin, sectioned (Leitz Rotary Microtome 1512, Leitz Tooling Systems Inc., Grand Rapids, MI, USA) and stained by standard hematoxylin and eosin (H\&E). Examination of H\&Estained sections was performed under light microscopy (Zeiss Axiophot 1, Zeiss Axiopath Systems, Carl Zeiss MicroImaging Inc., Thornwood, NY, USA) with $\times 2.5, \times 5, \times 10, \times 20, \times 40$ objective lenses.

\section{Confocal Microscopes}

For this study, two different near-infrared, reflectance confocal microscopes were utilized. For in vivo near-infrared, reflectance confocal microscopy studies, we used an experimental prototype designed in our laboratories, equipped with a $1054 \mathrm{~nm}$, Nd:YAG laser (neodymium:yttrium aluminum garnet, CV-4, Santa Fe Laser Company, Phoenix, AZ, USA) and $\times 60$ (0.85 NA, water-immersion 1UM571 Olympus, Lake Success, NY, USA) and $\times 30(0.9$ NA, water-immersion 96008 LOMO, Vermont Optechs, Charlotte, VT, USA) objective lenses. A detailed description of this system has been published elsewhere. ${ }^{2,3}$ In order to visualize dynamic images, the detector of this microscope is connected to a television monitor (Sony Trinitron), a VHS recorder setup (superVHS videotape recorder, Panasonic AG-7300) and a frame grabber of $8 \mathrm{bit} / \mathrm{pixel}$ (Pixelpipeline PTP425, Perceptics, Knoxville, TN, USA). For ex vivo near-infrared, reflectance confocal microscopy studies, a commercialized confocal microscope based on the experimental prototype previously described was used (VivaScope $2000^{\circledR}$, Lucid Inc., NY, USA), equipped with a $830 \mathrm{~nm}$, diode laser, a $\times 30$ lens and an inner computer 
setup. Importantly, the images obtained from both confocal microscopes used were equivalent in contrast and definition.

\section{Results}

\section{In Vivo Evaluation of Liver}

In vivo near-infrared, reflectance confocal microscopy images depict various tissue structures present in the most superficial layers of the liver. The hepatic capsule appears as a finely reticular, hyper-refractile mesh (Figure 1a). Under this mesh, the hepatic parenchyma displays a spongiform structure where hepatic cords are distinguished as rows of hepatocytes of moderate refractivity (gray on screen) (Figures 1-3). The parenchyma appears perfused by a series of vascular structures containing dynamic, hyper-refractile (white on screen) blood cells (Figures 1-3).

Hepatic plates appear as elongated, moderately refractile structures displaying a somewhat radial distribution around corresponding portal spaces and interconnecting between them. Within the plates, hepatocytes may be individualized as moderately refractile structures (gray on the images) with the nucleus appearing as a centered hypo-refractile
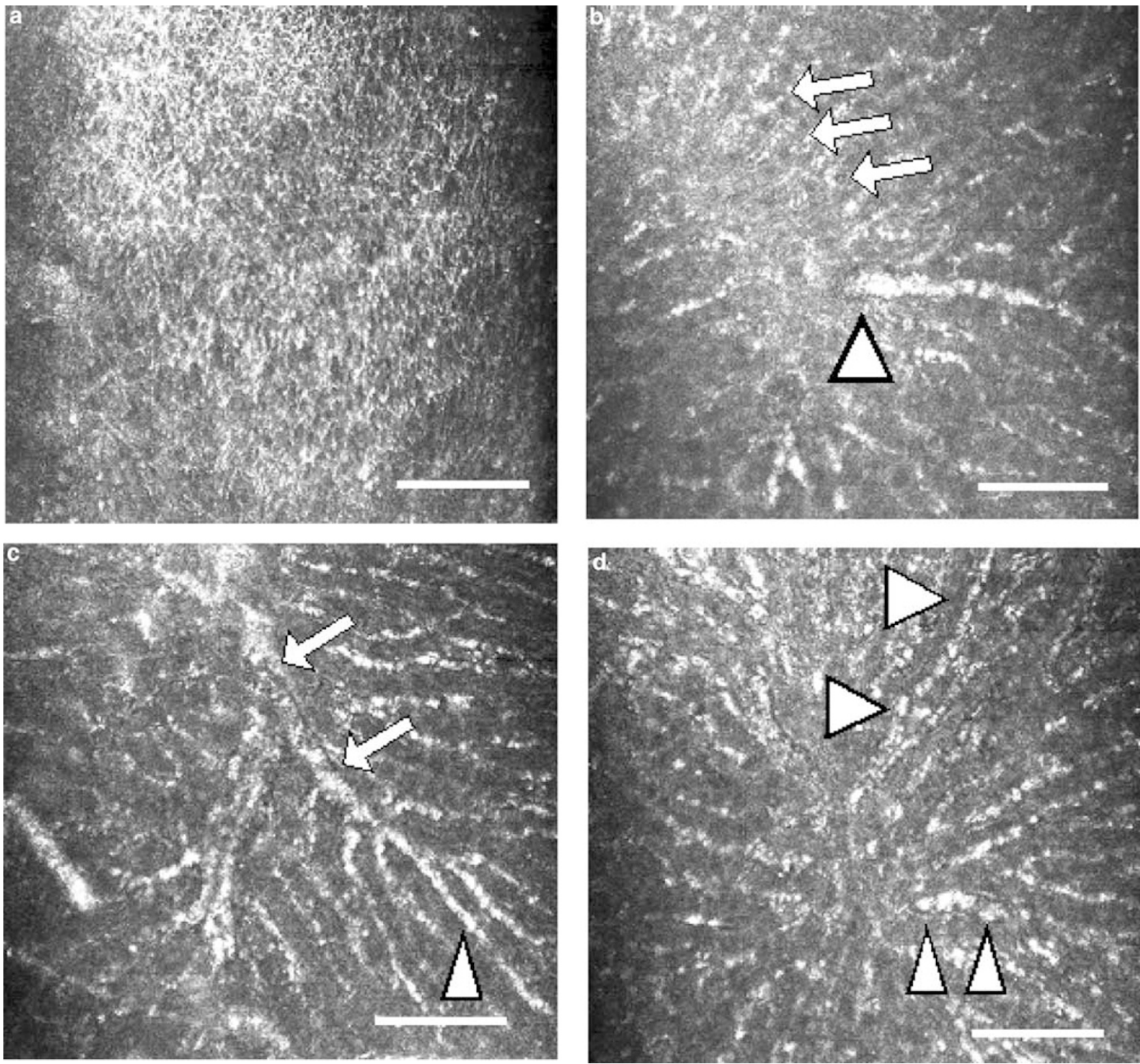

Figure 1 In vivo liver as seen with near-infrared, reflectance confocal microscopy. Beneath an intact capsule (a), the parenchyma is composed of moderately refractile, interconnecting hepatic plates (b-d). Hepatocytes are centered by round, hyporefractile areas corresponding to the nucleus (B, arrows). Fine hyporefractile canaliculi are seen in between hepatic plates (c, arrows). Highly refractile blood cells (white on screen) highlight the location of vessels (b and $\mathbf{d}$, arrowheads). Vascular structures seen here are either branches of central veins (b, arrowhead) or sinusoids (c, arrowheads). Scale bar represents $100 \mu \mathrm{m}$. 

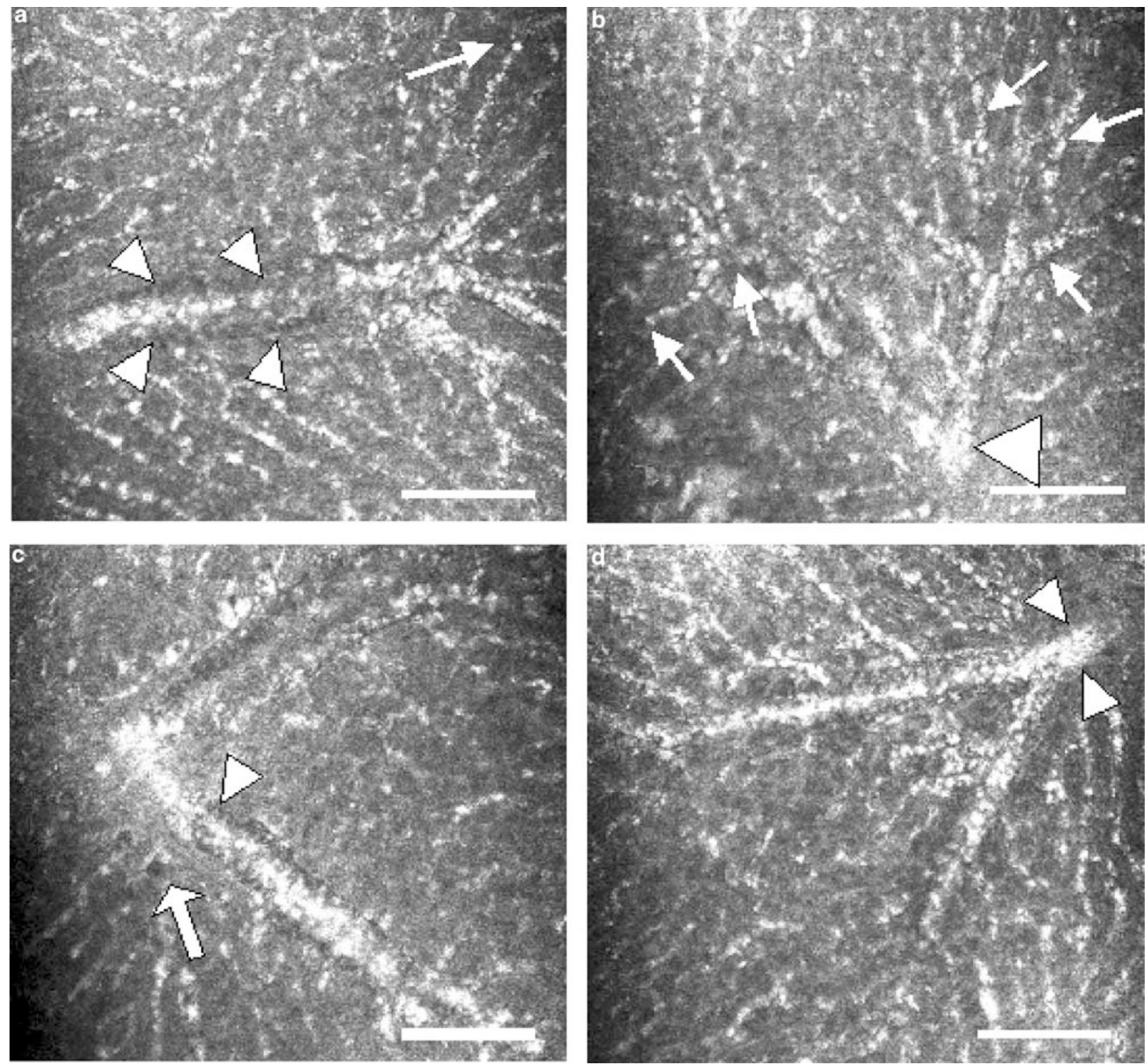

Figure 2 Major hyper-refractile vessels on the surface of liver in vivo correspond to branches of central/terminal veins (a-d, arrowheads). Vessels of smaller diameter, corresponding to sinusoids, may be tracked as they enlarge and drain into central veins. Hypoperfused vessels appear as a-refractile spaces (c, arrow). Near-infrared, reflectance confocal microscopy of serial planes provides information on the point at which the vessels pierce into deeper layers of the liver (c, arrowhead). The angles at which central veins branch out vary. Scale bar represents $100 \mu \mathrm{m}$.

spot (black on the images) inside which a hyperrefractile spot representing the nucleolus may be identified (Figure 1b). By contrast, the surrounding cytoplasm exhibits a slightly higher refractivity. The size of the hepatocyte ranges from 12.8 to $13.1 \mu \mathrm{m}$. No zonal variations in refractivity is appreciated among hepatocytes. Fine hyporefractile lines representing the canaliculi can be identified (Figure 1c). They appear along the hepatic plates, both in transversal (along the major axis) and in perpendicular directions. The hepatocyte's borders in direct contact with the canaliculi exhibit a greater refractivity. The limits of each hepatic plate appeared well-demarcated by the vessels (Figure 1d).
Under near-infrared, reflectance confocal microscopy examination, the vessels are easily visualized due to their dynamic and highly refractile blood content (Figure 2). Alternatively, plasma and vascular walls do not exhibit any contrast or refractivity, as appreciated when viewing a transverse section of a sinusoid devoid of blood (Figure 2c). Sinusoidal capillaries, postsinusoidal venules and branches of central veins may be identified throughout the superficial $300 \mu \mathrm{m}$ of the liver. Sinusoids are visualized as hyporefractile, branching channels ranging from 9.7 to $11.2 \mu \mathrm{m}$ in diameter (Figure 2a and b). Their size increases slightly when approaching the central veins into which they drain. 

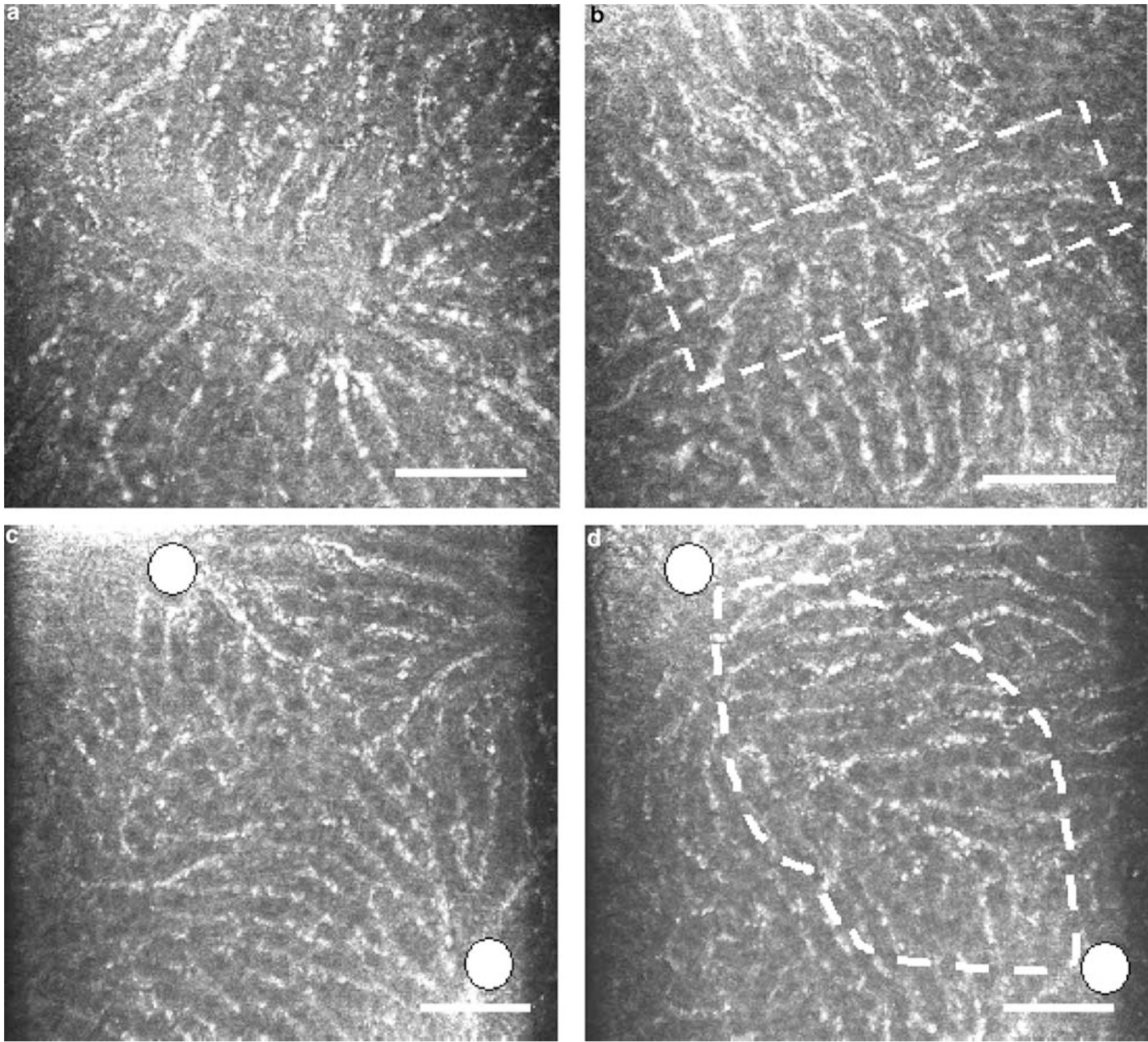

Figure 3 In in vivo near-infrared, reflectance confocal microscopy, the portal spaces lay below the superficial $300 \mu \mathrm{m}$ (a), being inferred out of a zone between sinusoids with opposite flow directions (b, rectangle). Central/terminal veins lay roughly equidistant and in opposite direction (c, $\mathbf{d}$, spheres). The oval-shaped area demarcated between central veins and centered by the portal zone corresponds to the hepatic acinus (d). Scale bar represents $100 \mu \mathrm{m}$.

Central veins measuring up to $46 \mu \mathrm{m}$ in diameter were seen on the surface of the liver in vivo (Figure 2). Three-dimensional tracking of these branches through near-infrared, reflectance confocal microscopy serial sectioning in real time shows how they drain the blood flow contributed by sinusoid capillaries and, eventually, pierce deep into the hepatic parenchyma (Figure 2c). The mean distance between two adjacent central veins was $555 \mu \mathrm{m}$.

Portal spaces lay more deeply in the parenchyma and hence are not directly visualized when performing near-infrared, reflectance confocal microscopy studies in vivo (Figure 3). Nevertheless, in the dynamic studies performed in real time, their situation could be inferred from the position of the corresponding central vein and the direction of the blood flow (Figure 3b). By exclusion, the periportal areas could be identified as stellate or ovoid areas from which sinusoids, with centrifuge blood flow, seem to emerge deep from the parenchyma extending towards the liver surface. Thus, the hepatic acini could be demarcated as the territories framed by two branches of central veins (Figure 3c) that host periportal areas on their equator (Figure $3 \mathrm{~d}$ ).

\section{Ex Vivo Evaluation of Liver}

Through the intact surface of the organ, nearinfrared, reflectance confocal microscopy imaging 

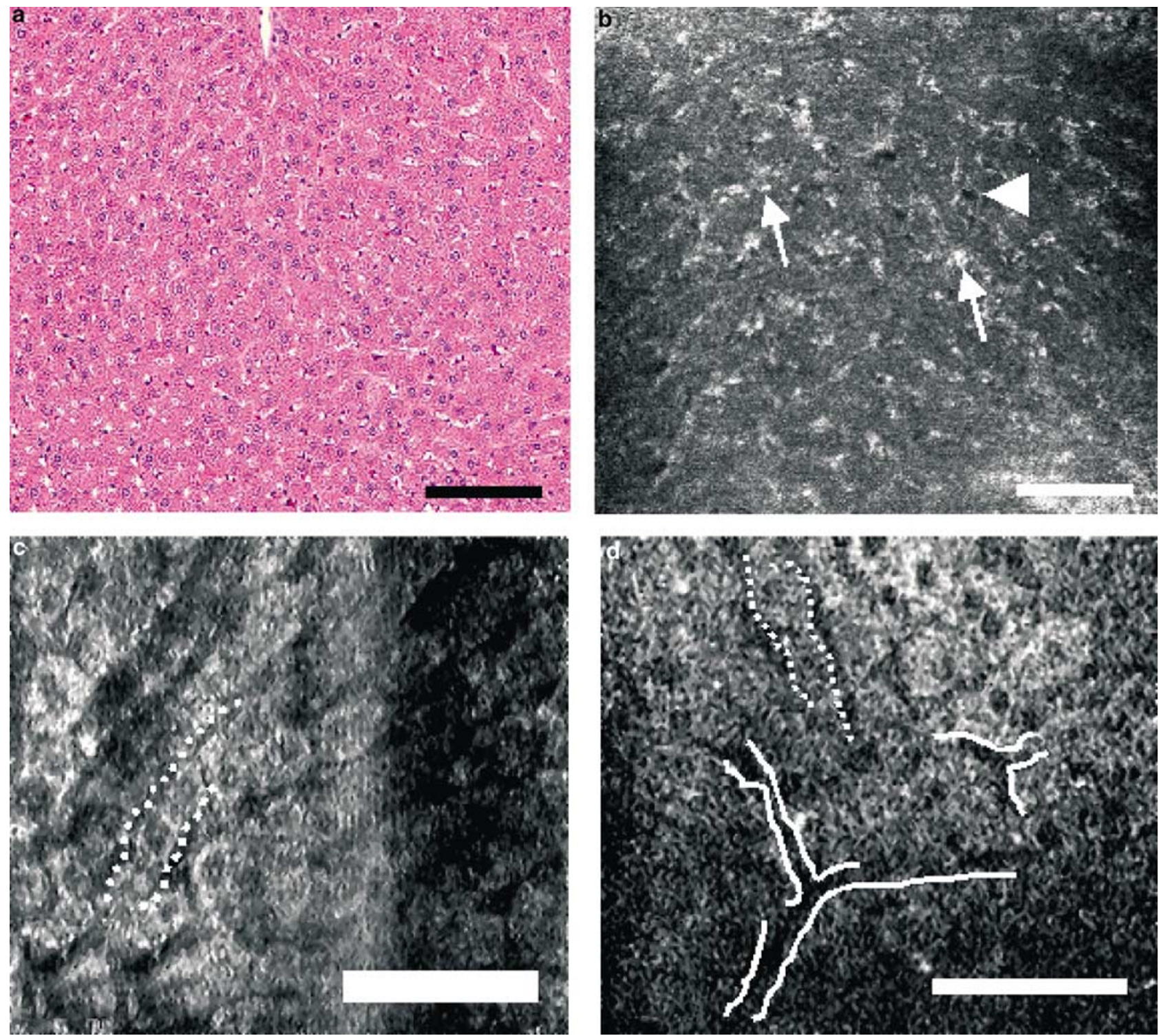

Figure 4 Ex vivo liver as seen with light microscopy $(\mathbf{a}, \times 10)$ and with near-infrared, reflectance confocal microscopy of freshly sectioned, unprocessed tissue $(\mathbf{b}-\mathbf{d}, \times 60)$. With a $830 \mathrm{~nm}$ diode laser, the hepatic plates display a moderate refractivity and lay in close contact making their individualization difficult (b). Vascular lumina are seen as round a-refractile areas (b, arrowhead) with occasional hyper-refractile erythrocytes trapped within (b, arrows). With an experimental $1064 \mathrm{Nd}$ :YAG laser, it is possible to demarcate and track hepatic plates (c and $\mathbf{d}$, bordered by discontinued lines). The hepatocytes appear as polygonal cells with a highly refractile cytoplasm and a hyporefractile nucleus centered by a hyper-refractile nucleolus (c). The sinusoidal tree is readily visualized as a network of arefractile channels (d, continued lines). Scale bar represents $100 \mu \mathrm{m}$.

of freshly sectioned livers allowed the visualization of parencyhma and vascular structures. Figure 4 illustrates the correlation between conventional light microscopic morphology of H\&E-stained sections and near-infrared, reflectance confocal microscopy of freshly sectioned unprocessed livers. The parenchyma exhibits a moderate refractivity and the vascular structures, now generally devoid of blood, appear as a-refractile spaces of various diameters.

Temporal structural changes are observed in the ex vivo tissue. In the early period (first minutes of ischemia), the hepatic plates preserve their size and general refractivity (Figure 5a). Occasionally, an hyper-refractile apical border may be distinguished (Figure 5a). Intracellularly, the nuclei may be identified as round structures of lesser refractivity with an occasional hyper-refractile nucleolus. Nearinfrared, reflectance confocal microscopy images feature both mononucleated and binucleated hepatocytes (Figure 5b). No zonal variation (central or periportal) of refractivity of the hepatic plates is seen. Progressively, homogeneization of the refractivity of the hepatic plates was noted making difficult the discrimination between structures (Figure 5b). The parenchyma gradually collapses, exhibiting a cobblestone-like pattern. 

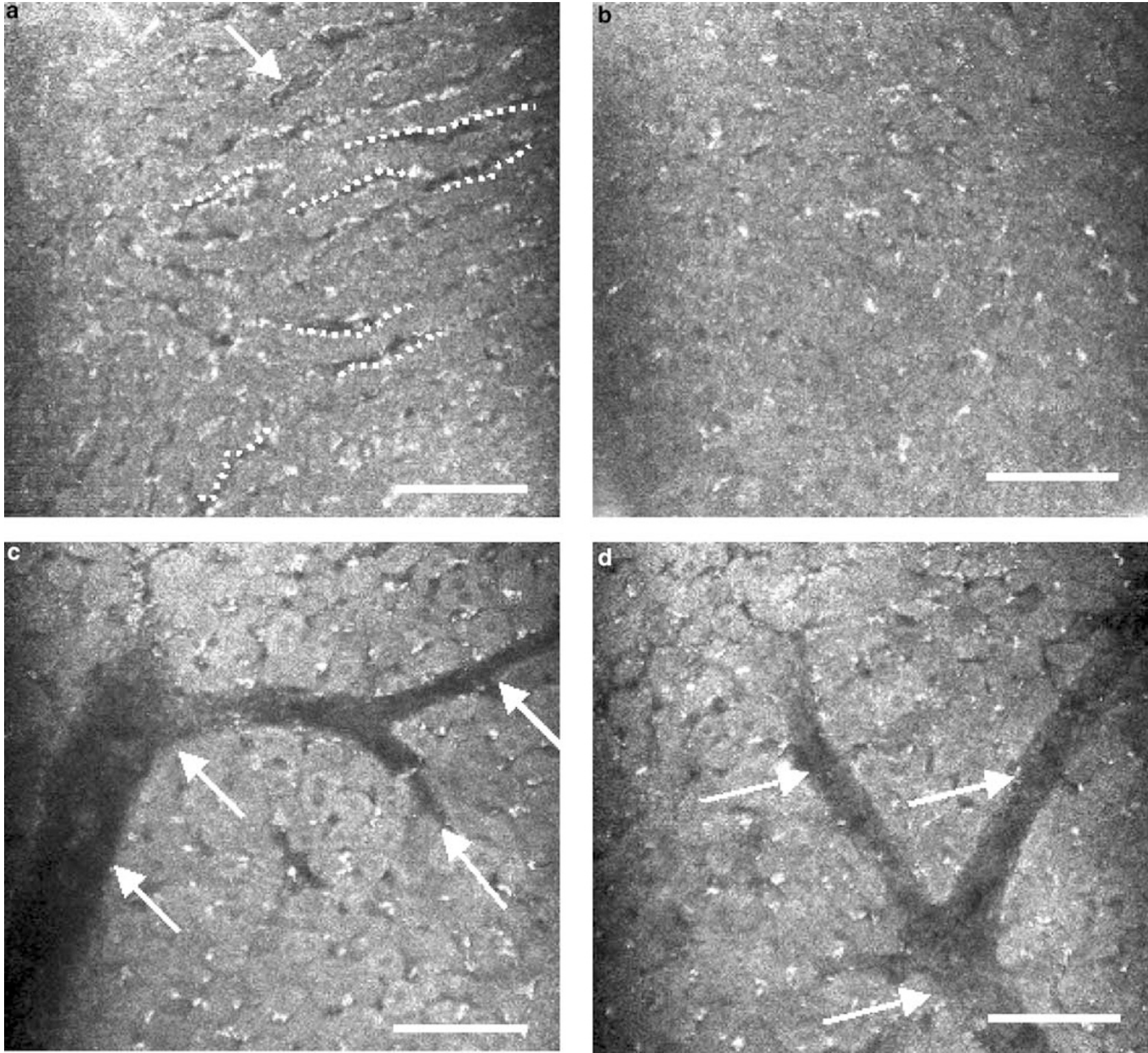

Figure 5 Vascular structures of rat liver ex vivo with near-infrared, reflectance confocal microscopy. Throughout the ischemic period, sinusoids tend to collapse and individualization of hepatic plates becomes difficult (compare (a) from an early ischemic period, to (b) of a later ischemic period). Hepatic plates ex vivo exhibit a moderate refractivity, occasionally displaying a hyper-refractile apical border (a, arrow). Sinusoidal spaces are a-refractile (a, discontinued lines). Greater sinusoids and branches of major vessels are readily observed as hyporefractile spaces giving branches that may be tracked (c and d, arrows). Scale bar represents $100 \mu \mathrm{m}$.

Sinusoids appear as very narrow hyporefractile channels (Figure 5a) generally devoid of blood except for occasional, trapped hyper-refractile cells. Larger sinusoids and major vascular structures are readily depicted as hyporefractile spaces giving branches that can be easily tracked (Figure 5c and d).

Sectioning of the liver in different planes allows imaging of structures that lay deeper than $300 \mu \mathrm{m}$ below the hepatic surface. Consequently, portal spaces and central veins with their large a-refractile lumina are readily identified (Figure 6). Within the portal spaces, portal veins and bile ducts can also be recognized (Figure 6a and b). Central veins are also depicted with clarity (Figure 6c and d).
Exposure of the hepatic tissue to experimental contrast agents induces selective changes in refractivity (Figure 7). Solutions of sucrose induce an increase in the contrast between the nucleus and cytoplasm of certain hepatocytes (Figure 7a and b). By contrast, exposure of the tissue to acetic acid solution increases the contrast of nuclei (Figure 7c and d).

\section{Discussion}

This is the first microscopic study on animal liver performed in vivo, in real time, in a nondestructive 

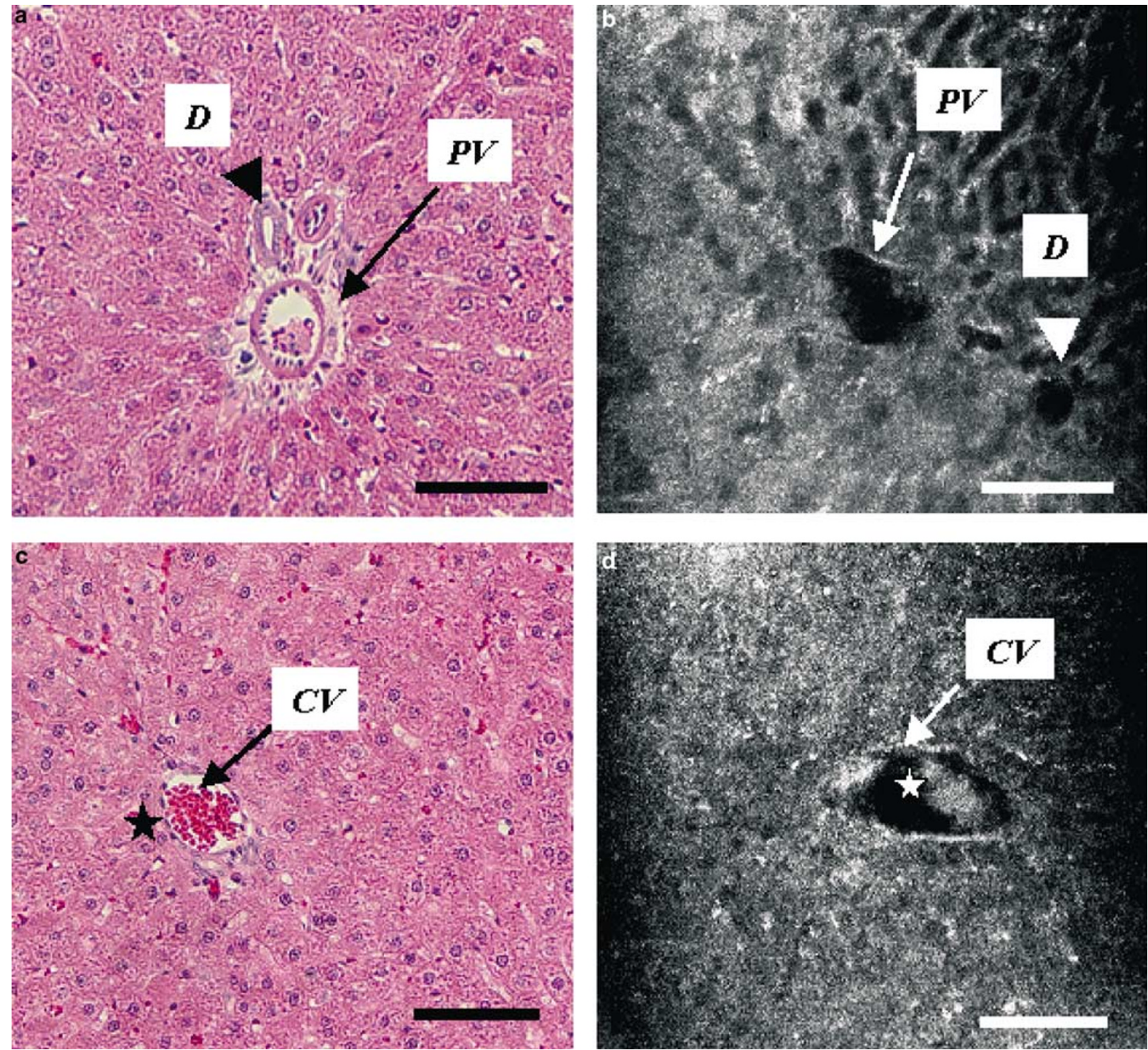

Figure 6 Ex vivo rat liver featuring a portal space and a central/terminal vein, as seen on H\&E sections $(\mathbf{a}$ and $\mathbf{c}, \times 10)$ and in freshly sectioned tissue with near-infrared, reflectance confocal microscopy (b and $\mathbf{d}$ ). The portal space (a and $\mathbf{b})$ includes a branch of portal vein $(P V)$ and the biliary duct $(D)$. The central vein (c and d, $C V$ ) contains trapped blood (c and d, stars). Scale bar represents $100 \mu \mathrm{m}$.

manner and without any exogenous agent. It demonstrates that near-infrared, reflectance confocal microscopy is a useful imaging technique that identifies key histological hepatic structures. Subcellular details like the location and number of nuclei can also be assessed without exogenous agents, avoiding photobleaching or leakage through sinusoidal fenestrations, frequent inconveniences of fluorescent agents. Near-infrared, reflectance confocal microscopy images of freshly sectioned liver show an excellent correlation with the morphology of H\&E-stained sections examined under standard light microscopy. One advantage of near-infrared, reflectance confocal microscopy is that it, apparently, does not alter the integrity of the tissues, allowing further experimentation using the same specimen with other techniques. Interestingly, differences in architecture and refractivity patterns were shown between in vivo and ex vivo rat livers.

This work demonstrates that near-infrared, reflectance confocal microscopy visualizes essential architectural elements such as hepatic plates, sinusoids, venules and portal spaces. However, defining the limits of the basic parenchymal unit was not possible because in vivo near-infrared, reflectance confocal microscopy cannot properly depict portal spaces or recognize differences in refractivity between hepatocytes of different acinar zones. Part of the difficulty lays also in the fact that, in the rat liver, basic functional units are not 

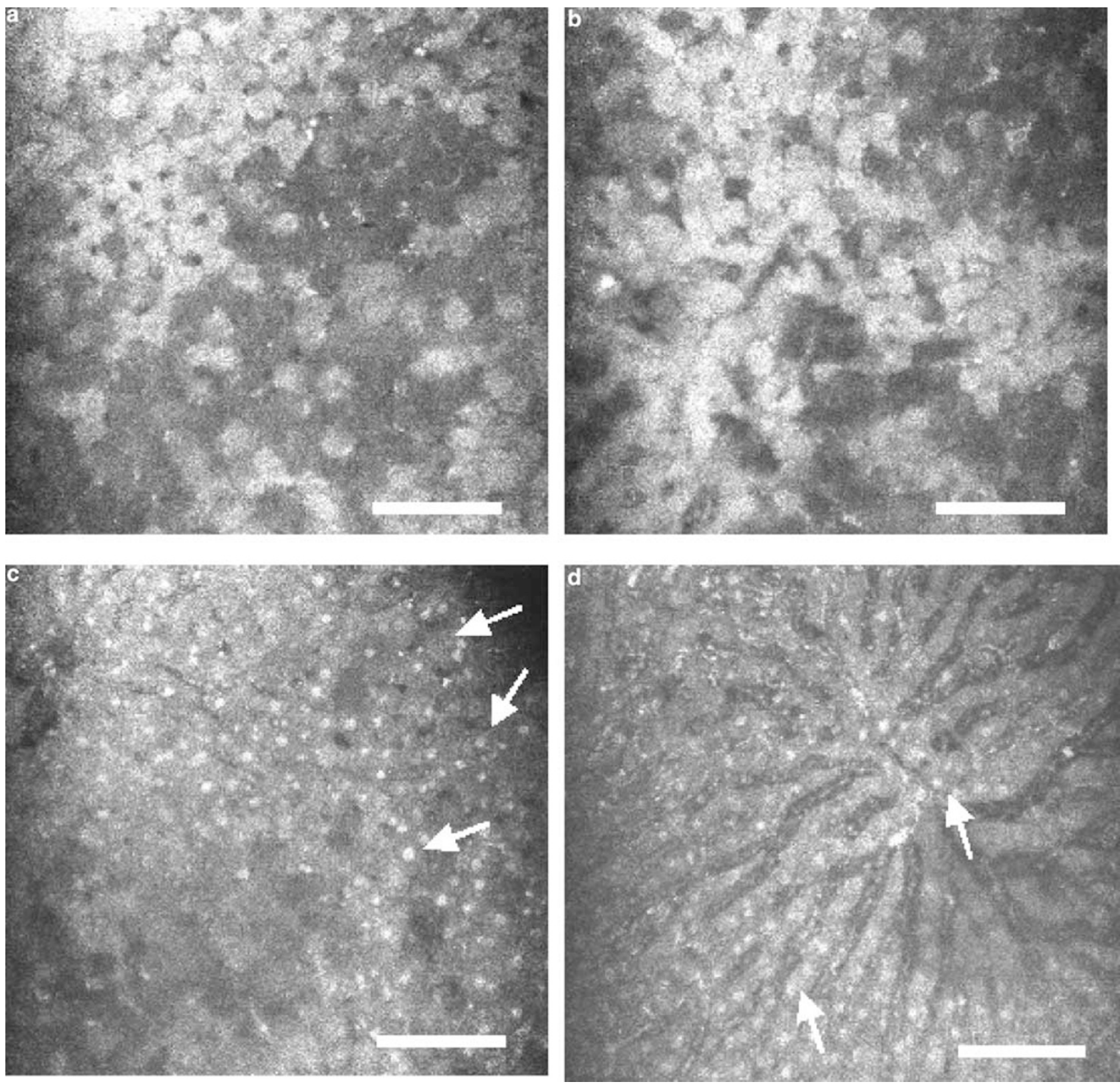

Figure 7 Near-infrared, reflectance confocal microscopy imaging normal ex vivo rat liver, exposed to contrast agents. Exposure to sucrose highlights specific hepatocyte populations (a and $\mathbf{b}$ ). Exposure to acetic acid renders nuclei more refractile, appearing as white spots (c and d, arrows). Scale bar represents $100 \mu \mathrm{m}$.

separated by connective strands as they are in the pig. Many studies have tried to define the hepatic unit, both structurally and functionally, using either different techniques of intravital microscopy ${ }^{4-7}$ or reconstructions of ex vivo, injected specimens. ${ }^{8-10}$ Ex vivo studies of fibrosed livers suggest that the basic unit would be a unit centered by a portal triad, from where the blood would flow toward hepatic veins located in the periphery, ${ }^{8}$ implying an organization exactly the opposite to the pig and human livers. Actually, enzymatic studies in ex vivo liver suggest that neither the acini nor the portal units truly define the basic organization of the rat liver, and accordingly, the basic unit would have the shape of a cone, with branches of terminal hepatic vein in its center and portal triads at the periphery. ${ }^{11}$

Most in vivo imaging studies convey that in the rat sinusoids and branches of central veins are located on the surface, while their corresponding portal branches lay at a greater depth. This histologic anisotropy contributes to the fact that some structures of the hepatic unit cannot be visualized in vivo with epi-illumination fluorescence microscopy. ${ }^{7}$ Since central/terminal hepatic vein branches can be seen under near-infrared, reflectance confocal microscopy, we took Rappaport's acinus as a 
reference for describing our findings. This model establishes a series of landmarks or zonal frontiers among hepatocytes, dividing them according to their disposition along a blood gradient. ${ }^{12}$ The vertices of the acinus are branches of central veins and its equator is the communication between two adjacent portal triads. However, under near-infrared, reflectance confocal microscopy no zonal differences in refractivity has been observed in vivo.

The use of near-infrared, reflectance confocal microscopy currently faces a number of limitations including the limited depth of imaging within the organ and the lack of sharpness in the evaluation of intracellular detail. Near-infrared, reflectance confocal microscopy images, though, show that the nucleus and cytoplasm exhibit different refractivity and the possibility of labelling nuclei in nearinfrared, reflectance confocal microscopy remains an interesting option. Acetic acid is believed to induce conformational changes in the nuclear chromatin with release of histones and formation of larger DNA aggregates $(1-5 \mu \mathrm{m})^{2,3}$ that become more refractile. ${ }^{13-15}$ Importantly, acetic acid does not change morphologic diagnostic information and may be safely used for confocal microscopy studies prior to routine histologic processing. ${ }^{2,3}$ However, citric acid, another nuclear labelling agent used in near-infrared, reflectance confocal microscopy, has been associated with alterations in the viability, adhesion and protein synthesis of cultured cells induced by extracellular acidosis. ${ }^{16}$ An association between acetic acid and induction of apoptosis has also been demonstrated. ${ }^{17}$ Therefore, investigations on the underlying mechanisms of the contrast agents used in this study (acetic acid and sucrose solutions) and their safety are justified before usage in clinical research.

Currently available setups allow the evolution of the most superficial $200-300 \mu \mathrm{m}$, yielding observations that may not be representative of the deeper morphology. However, since the accumulation of white blood cells and their interaction with the endothelium takes place mainly in postcapillary vessels seen on the hepatic surface, near-infrared, reflectance confocal microscopy could be a valuable tool for the imaging of inflammatory processes. In vivo evaluation of hepatic microcirculation can also be beneficial to the understanding of sinusoidal constriction during hepatic reperfusion and postclamp ischemia, frequent complications of hepatic surgery. Near-infrared, reflectance confocal microscopy has already been proven useful in studying characteristic pathologic patterns present in human liver, particularly fatty deposits and fibrosis. ${ }^{18}$

In summary, near-infrared, reflectance confocal microscopy is a novel imaging technique that provides morphologic information of tissues both in vivo and ex vivo. With technical improvements and the development of new applications, nearinfrared, reflectance confocal microscopy could become useful to surgeons and pathologists for rapid functional and morphologic assessment of hepatic parenchyma during surgical interventions or histopathologic evaluation. With technical improvements, such as the development of a fiber optic version of a confocal microscope,${ }^{19}$ one can envision the utilization of near-infrared, reflectance confocal microscopy by hepatologists, surgeons and pathologists for rapid functional and morphologic assessment of hepatic parenchyma preoperatively or during surgery.

\section{Acknowledgements}

We would like to thank Mr John $\mathrm{T}$ Demirs for preparation of histology sections, and Mr Stephen A Conley for assistance in histology slide photography. This work was partially funded by Lucid Inc (Rochester, NY, USA).

\section{References}

1 Pawley J (ed). Handbook of Confocal Microscopy, 2nd edn. Plenum Press: New York, 1995.

2 Rajadhyaksha M, Grossman M, Esterowitz D, et al. In vivo confocal scanning laser microscopy of human skin: melanin provides strong contrast. J Invest Dermatol 1995;104:946-952.

3 Rajadhyaksha M, González S, Zavislan JM, et al. In vivo confocal scanning laser microscopy of human skin II: advances in instrumentation and comparison with histology. J Invest Dermatol 1999;113:293-303.

4 Kan Z, Ivancev $\mathrm{K}$, Lunderquist $\mathrm{A}$, et al. In vivo microscopy of hepatic tumors in animal models: a dynamic investigation of blood supply to hepatic metastases. Radiology 1993;187:621-626.

5 Kawano K, Bowers JL, Kruskal JB, et al. In vivo microscopic assessment of hepatic microcirculation during liver allograft rejection in the rat. Transplantation 1995;59:1241-1248.

6 Mc Cuskey RS, Vonnahme FJ, Grun M. In vivo and electron microscopy observations of the hepatic microvasculature in the rat following portacaval anastomosis. Hepatology 1983;3:96-104.

7 Menger MD, Marzi I, Messmer K. In vivo fluorescence microscopy for quantitative analysis of the hepatic microcirculation in hamsters and rats. Eur Surg Res 1991;23:158-169.

8 Bhunchet E, Wake K. The portal lobule in rat liver fibrosis: a re-evaluation of the liver unit. Hepatology 1998;27:481-487.

9 Teramoto K, Bowers JL, Kruskal JB, et al. Hepatic microcirculatory changes after reperfusion in fatty and normal liver transplantation in the rat. Transplantation 1993;56:1076-1082.

10 Yamamoto K, Sherman I, Phillips MJ, et al. Threedimensional observations of the hepatic arterial terminations in rat, hamster and human liver by scanning electron microscopy of microvascular casts. Hepatology 1985;5:452-456.

11 Teutsch HF, Schuerfeld D, Groezinger D. Three dimensional reconstruction of parenchymal units in the liver of the rat. Hepatology 1999;29:494-505. 
12 Mc Sween RNM, Desmet VJ, Koskaws T, et al. Developmental anatomy and normal structure. In: McSween RNM, Burts AD, Portmann BC, KG Ishak, Schever PI, Anthony PP (eds). Pathology of the Liver, 4th edn. Churchill Livingstone: New York, 2002.

13 Drezek RA, Collier T, Brookner CK, et al. Laser scanning confocal microscopy of cervical tissue before and after application of acetic acid. Am J Obstet Gynecol 2000;182:1135-1139.

14 Fraschini A, Pelliciari C, Biggiogera M, et al. The effect of different fixatives on chromatin: cytochemical and ultrastructural approaches. Histochem J 1981; 13:763-769.

15 Smithpeter C, Dunn A, Drezek R, et al. Near real-time confocal microscopy of cultured amelanotic cells: sources of signal, contrast agents and limits of contrast. J Biomed Opt 1998;3:429-436.

16 Lan WC, Lan WH, Chan CP, et al. The effects of extracellular citric acid acidosis on the viability of extracellular adhesion capacity and protein synthesis of cultured human gingival fibroblasts. Aust Dent J 1999;44:123-130.

17 Ludovico P, Sousa MJ, Silva MT, et al. Saccharomyces cerevisiae commits to a programmed cell death in response to acetic acid. Microbiology 2001;147:2409-2415.

18 Campo-Ruiz V, Ochoa ER, Lauwers GY, et al. Human liver biopsy studied with near-infrared confocal microscopy. A pilot study. Hum Pathol 2002;33:975-982.

19 Tearney GJ, Webb RH, Bouma BE. Spectrally encoded confocal microscopy. Opt Lett 1998;23:1152-1154. 